\title{
Conference Summary
}

\author{
Peter N. Wilkinson \\ University of Manchester, Jodrell Bank Observatory, Macclesfield, \\ CHeshire, Sk11 9DL UK
}

Abstract. A personal view of the conference highlights.

\section{Introduction}

IAU205 provided an all-too-rare opportunity for our wavelength-chauvinist communities to share the latest high resolution results and to tell each other about plans for future telescopes. The timing was right, since Chandra has produced its first flush of spectacular images, and the place was right since it was Manchester astronomers who laid some of the foundations of high angular resolution techniques, both in the radio and the optical. Manchester continues its high resolution tradition by operating the MERLIN radio array.

To get my excuses in right at the beginning, I contend that the summariser's task for this invigoratingly varied symposium of 61 talks and 105 posters, which touched upon most aspects of astrophysics, is tougher than usual! Nevertheless, as well as noting some of the stand-out results, I have tried to provide some broad impressions of the present observational landscape.

First, then, some broad-brush comments about instrumental capabilities. As expected we saw many high quality results from the current generation of radio and $\mathrm{mm}$-wave interferometers; high resolution radio imaging is a longsolved problem. There was plenty of new and exciting data from the current crop of $8 \mathrm{~m}$ class optical/infrared telescopes especially sub-arcsec imaging in the nearIR ( $\mathrm{K}$ to 15 microns) band. And our high hopes for Chandra's sub-arcsecond $\mathrm{X}$-ray imaging were more than fulfilled. But there were some disappointments, in particular we saw very few results from optical/infrared adaptive optics or interferometry. The conclusion must be that both techniques are not yet mature enough to make general-purpose contributions to astrophysics. Now for my observer's eye view of the scientific highlights from the meeting.

\section{Galaxies and their inner regions}

\subsection{The compact mass in the Galactic Centre}

Year-by-year observers are steadily zeroing in on the characteristics of the mass in the Galactic Centre (GC). A striking movie made from multi-epoch speckle-imaging data, showed statistically Keplerian stellar motions consistent with a central point mass of $3 \times 10^{6} \mathrm{M}_{\odot}$. Newly-detected orbital accelerations of the

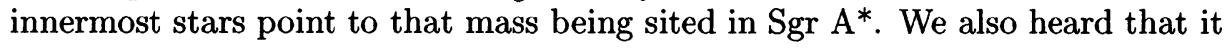


was feasible with interferometry to measure periastron motions for such stars. Prograde motion would be a diagnostic of general relativistic effects and its detection would essentially prove the existence of a central black hole. In the radio band we heard that $\mathrm{mm}$-wave VLBI of the GC is already probing scales of 15 Schwarzschild radii and in the future sub-mm VLBI could probe the event horizon itself.

\subsection{Compact masses in external galaxies}

In other disk galaxies the central regions are usually obscured and it is hard to get reliable velocity signatures since gas motions may be confused by circumnuclear mass clumps and starbursts. In nearby ellipticals, however, the central stellar regions are more accessible and a major industry has grown up, based on the HST, which is revealing the ubiquity of compact (but only on 100pc scales) central masses via photometry of central light cusps and measurement of their velocity dispersions. The present evidence seems to be consistent (but does not prove) that all galaxies contain massive black holes, whose mass scales with that of the bulge. Any such global correlation between such different physical scales could not be expected a priori and suggests that $\mathrm{BHs}$ and galaxies may form together.

An important related question is: how many AGN are obscured in galaxies, particularly at high $\mathrm{z}$, by dust? The host galaxy of the well-known radio-loud quasar 3C48 contains a powerful jet-driven starburst whose SED shows a very similar infrared dust bump to that exhibited by classical starbursts/ULIRGs. How many more of these hidden AGN are there amongst more distant, less radio luminous, objects? The detection of compact radio structure is a particularly simple diagnostic of an AGN and, as sensitivities rise, radio imaging is having an increasing impact on the AGN/starburst dichotomy. For example VLA/MERLIN and European VLBI imaging of the Hubble Deep Field reveals that most of the radio detections down to tens of microJy are associated with starbursts but there are more AGN than was expected ( $>20 \%$ in the Deep and Flanking Fields). We also heard that a combination of the IRAM interferometer and the VLA/MERLIN imaging (the latter providing the vital astrometry to link the HDF frame to the IRCF frame) has shown that the brightest SCUBA source in the HDF is not optically identified down to the HDF limit! The most likely explanation is that this is a very heavily dust obscured galaxy.

\subsection{Super-high brightness temperatures in AGN}

A convincing case was made that the perplexing intraday variability (IDV) observed in compact radio sources is primarily due to interstellar scattering (ISS). Two objects have been seen to double or triple their flux in 1 hour and the implied brightness temperatures of $\sim 10^{21} \mathrm{~K}$ are simply too bright to be intrinsic, even allowing for relativistic beaming. There is more direct evidence in favour of ISS in these objects since a 2-minute time delay between variations seen at Parkes and at the VLA has been seen in PKS 0405-385 while seasonal variations in variability have been seen in $\mathrm{J} 1819+385$. However this does not leave us problem-free. In order to exhibit ISS the angular sizes must be $<10$ microarcsec and the brightness temperatures must be $\sim 10^{14.5} \mathrm{~K}$. These temperatures are still rather high since the directly-measured (by VLBI) Lorentz 
factors in compact sources are generally too low to boost an intrinsic $10^{11.5} \mathrm{~K}$ to $10^{14.5} \mathrm{~K}$. This dichotomy should, perhaps, not worry us too much since these are almost certainly amongst the most extreme objects. Of more concern is the fact that there seem to be too many strong sources $(\sim 25 \%)$ which show IDV. New Space VLBI results from the VSOP mission provide independent evidence for temperatures $>10^{12} \mathrm{~K}$ in $50 \%$ of the sources studied. Are these high fractions of bright nuclei explicable solely with beaming or could we be seeing the first evidence of coherent emission in AGN?

\subsection{Circumnuclear tori}

The broad picture of a central mass surrounded by some sort of disk or torus is now generally accepted and in the last five years radio absorption line studies have begun to reveal details of the inner regions. In three radio galaxies VLBI has detected free-free absorption against a radio counter-jet and by this means has begun to probe the ionized central pc. In nearby Seyferts the evidence suggests an onion skin structure of different atomic and molecular species. The outside skin, on $1 \mathrm{kpc}$ scales, consists of $\mathrm{HI}$ which has also become accessible to radio absorption studies. The inferred disk properties are consistent with HST pictures of dust disks at much lower resolution. However, the radio jets are not always aligned to the dust disks and thus the spin axis of central $\mathrm{BH}$ does not always know about the overall galactic rotation axis.

\subsection{Supernovae in external galaxies}

Estimating the massive star death rate, by counting the number of SNR, is a pretty direct measure of the massive star birth rate in starburst galaxies. For determining overall star formation rate (SFR) one needs a model of the IMF but this is a complementary approach to those based on measurements of the integrated FIR or radio continuum emission. M82 is a particularly accessible laboratory for SNR studies but only in the radio band where we can see through the dust. A fine VLA/MERLIN radio image shows up to 50 individual SNR; some of these have been seen to expand by means of VLBI imaging and ages 30 to 1000 years have been inferred for different remnants. Reassuringly for M82 all three "favoured" methods of obtaining the SFR appear to be consistent. Another boost in radio sensitivity would allow these SNR-counting techniques to be applied to a wide range of nearby starbursts.

At greater luminosities Arp 220 is the archetypical ULIRG but its $\sim 1000$ magnitures of visual extinction provide a massive impediment to detailed study in any band other than the radio. Global VLBI imaging has so far failed to locate an AGN but has revealed about 12 compact sources, which are almost certainly SNR, dotted about in the western condensation. These SNR have much higher luminosities than virtually all those in M82; they do however, share the luminosity of the brightest remnant in M82 $(41.9+58)$ which has some peculiar properties. Monitoring observations from 1994 to 2000 suggest an SNR rate of $\sim 0.4$ /year, significantly lower than expected if supernovae are the sole power source of Arp 220. But the rate is so far based on small number statistics and presumably there is a missing population of low luminosity SNRs, like those in M82, still to be seen. VLBI observations of Arp 220 have a great future, but a long way to go. 
The shell development of SN1993J has received particularly close attention from the VLBI community. In a tour-de-force of VLBI imaging two groups have made independent multi-epoch data into two beautiful movies. Broadly the expansion is circularly symmetric and by the time of the meeting, deceleration to half the original velocity had been measured. The progenitor could be a binary due the low inferred mass loss rate.

It is clear that there is a wide range of phenomenology in young extragalactic SNR and, as usual, we need more and better data to disentangle the real systematics from the peculiarities induced by the local environment into which each remnant expands.

\subsection{X-ray imaging of external galaxies}

For many of us either living outside the USA, or not in the X-ray community, this meeting was the first chance to see many of the new results being produced by Chandra. Its resolution, corresponding to $<100 \mathrm{pc}$ in nearby galaxies, provides a dramatic imaging improvement over ROSAT. The typical Chandra image of a nearby galaxy shows a set of "hard" discrete sources, similar in properties to Galactic XRB sources (radiating at the Eddington luminosity for a solar mass neutron star), embedded in "soft" diffuse halos associated with star forming regions. But Chandra is also finding a new super-bright population of highly-variable XRB exhibiting luminosities hundreds of times the norm. One particular, very-hard-spectrum, source in M82 is radiating $\sim 10^{41} \mathrm{ergs} \mathrm{sec}^{-1}$ which corresponds the Eddington luminosity for $\sim 500 \mathrm{M}_{\odot}$. This source lies at the position of a radio transient and its nature is an exciting mystery.

With the best test case being M82, there appears to be no strong tie up between $\mathrm{X}$-ray sources and compact radio sources. This is perhaps not surprising since the latter are dominated by young SNR rather than XRB. It seems that the the radio is telling us about the dead high-mass stars while the X-rays tell the story of the living dead-one component of an XRB being a highly evolved object!

\subsection{A stellar supercluster}

One of the clearest cases where the "value-added" power of multi-wavelength data was apparent, involved the radio and mid-IR detections of a super-star cluster in a dwarf galaxy. The comparable resolution of the VLA and the Keck (12 micron) diffraction-limited image at 0.25 arcsec, provides the key to an interpretation involving radiation from 4000 O7 stars; in turn this suggests that we are looking at the precursor of a globular cluster where 1 million stars lie within $\sim 2$ parsec.

\subsection{Molecules in External Galaxies}

We were reminded of the distinction between the two main species of megamasers. The $\mathrm{OH}$ masers occur in exclusively in ULIRGS and starbursts galaxies pumped by stellar FIR but the systematics of these megamasers is far from clear. Water masers on the other hand, are invariably associated with gas in circumstellar disks in AGN and can therefore be used to determine the central masses, as in case of NGC4258. Sadly we learnt that the discovery rate for new systems 
is low, only 21 out of 350 galaxies searched. Despite many years of study the intrinsic physics of the both types of masing regions remains poorly understood.

The existing mm-wave arrays (OVRO, BIMA, IRAM) are producing some spectacular images of nearby galaxies, which provide unique constraints on the overall kinematics and the physical and chemical state of the thermal gas. The combination of $\mathrm{CO}$ imaging together with radio continuum and ISO imaging allows us to cut through the heavily obscured starbursting regions. Arp 220 provided a particularly fascinating result-the merging nuclei are counter-rotating. Millimetre wave imaging has just started to reach out to AGN and in the first imaging of a low-z quasar, $1 \mathrm{Zw1}$, a $1 \mathrm{kpc}$ molecular ring around the nucleus has been detected. Clearly we are just seeing the tip of an iceberg which will be visible to ALMA.

\section{Observations of Galactic Objects}

\subsection{Timing and fundamental physics}

I suspect that many of the audience shared my unfamiliarity with the power of high temporal resolution for exploring fundamental physics. In particular we heard about the Rossi X-ray timing Explorer and how observations of soft $\mathrm{X}$-ray repeaters, which involve pulsed emission from regions with very strong magnetic fields $\left(\sim 10^{15}\right.$ gauss), present a great opportunity to look for QED effects. In addition the high Q (about 1000) quasi-periodic oscillations (QPOs) with frequencies of $\sim 1 \mathrm{kHz}$ can be identified with a general relativistic effectrotation at the last stable orbit around a black hole.

Unfortunately the scales probed by such X-ray timing is far smaller than that probed by any direct measurement and so these techniques mostly have to stand alone. There is, however, a useful link with other physical diagnostics in galactic micro-quasars which produce relativistic radio-jets during outbursts, often when the X-rays indicate that the accretion disk has temporarily collapsed. In such objects we heard that the QPOs have been associated with disk oscillations and inertial frame dragging-again general relativistic effects depending on the mass and spin of hole.

There was very little about pulsar timing (there are lots of specialist pulsar meetings!) which has long been used as a probe of relativistic effects-as witness the award of a Nobel Prize. We did, however, hear of a case where a different binary pulsar shows effects expected from general relativistic geodetic precession. My previously half-closed eyes have been opened to the power of timing!

\subsection{Masers in a Stellar Photosphere}

Moving on to normal stars, one of the visual highlights of the meeting was a beautiful movie from the VLBA showing the varying positions of $\mathrm{SiO}$ masers in the photosphere of a red giant star (TX Cam) with milliarcsec resolution. Here the full power of the VLBA has been harnessed to produce unique, direct, insights into the conditions in a stellar photosphere other than the Sun's. It is clear that the mass outflow is non-symmetric and that the dynamics are probably dominated by shocks near the stellar surface. There is very clearly potential for much more fruitful work for VLBI in this area. 


\subsection{Optical imaging of stars}

Rather disappointingly we heard few new results on stellar imaging from optical Michelson interferometers, although it was impressive to hear the reports of the quasi-industrial scale of the developments at the VLT and the Keck sites. The current state of the art is that angular diameters of giant/hot stars can be obtained to a few percent but more subtle measurements, such as limb darkening, are much harder and there are currently only a few clear-cut results. $\mathrm{TiO}$ aborption in the atmospheres of giant stars can give rise to particularly marked changes in their apparent diameter with wavelength. Measuring the pulsations of Mira and Cepheid variables is, however, now on the horizon and one sigma results have now been claimed on a Cepheid. Direct imaging of stellar photospheres remains limited, although it has been clearly established that Betelegeuse shows deviations from uniform brightness. It seems we are nowhere near making optical images to match the SiO maser images of TX Cam. We await results, particularly from the VLTI and Keck interferometers, with keen anticipation.

\subsection{Chandra imaging of supernovae}

We saw spectacular Chandra spectral images of Galactic SNR shells which provide powerful insights into elemental differentiation, models of the progenitor core and the processes of explosive nucleosynthesis. An onion-skin model fits the results pretty well and in one remnant there are eight solar masses of oxygen! Non-thermal emission identified in SNRs could well be the site of cosmic ray acceleration. These SNR results are already another triumph for Chandra.

\subsection{Star formation}

Star formation is one topic which demands a combination of multi-wavelength data and high resolution to make real progress. The earliest epochs in the formation process $\left(10^{4}\right.$ years) are obscured and hence can best be studied at radio and (sub-)mm wavelengths. The mid-epoch $\left(10^{5}\right.$ to $10^{6}$ years) can also be studied in the mid-ir while the epoch of planet formation $\left(10^{7}\right.$ to $10^{8}$ years) is the optically-revealed phase. We are, however, a long way from being able to see the "big-picture" but at least we saw some beautiful new results. For example near-ir images in molecular hydrogen at 2.1 microns reveal motion at tens of $\mathrm{km} / \mathrm{sec}$ in the jets of Herbig-Haro objects. We also saw superb JHK images of the Orion GMC with ISAAC camera on the VLT at 0.5 arcsec resolution and with the Calar Alto 5 micron camera. This new mid-ir imaging capability on large telescopes was most impressive. Very close to the star, however, the radio band will long remain the only option with $\mathrm{SiO}$ and water masers often delineating outflows and methanol masers often providing high brightness tracers of rotating disks. To link the larger dust disks, such are those seen in absorption with the HST in Orion, to the scales probed by radio one needs to resolve scales of a few $\mathrm{AU}$ in other wavebands i.e. $0.01 \mathrm{arcsec}$ at Orion. This is an area where the VLT and Keck interferometers can make a major impact. 


\section{New Telescopes for High Resolution}

\subsection{Radio to sub-millimetre}

In the second half of this decade the optical communications revolution will allow cost-effective fibre-based upgrades of both the VLA (EVLA) and MERLIN (e-MERLIN) and VLBI. The hugely increased data transfer rate will provide factors of 10 to 30 increase in the sensitivity of these already highly-successful instruments.

The complex technique of space VLBI has now been proved by the successful Japanase-led VSOP imaging mission. The Russian-led RADIOASTRON mission, awaiting a launch at an unspecified date, is aimed at very high orbit and hence could search for very high brightness temperatures indicative of coherent emission. The next generation of missions (Japan's VSOP2 and the USA's ARISE ) are now in the planning stage and would not only provide 10-100 times more sensitivity than VSOP at $\mathrm{cm}$-wavelengths but at $\mathrm{mm}$-wavelengths would probe scales comparable to the last stable orbit around a black hole. VSOP2 could be launched in 2008.

The Square Kilometre Array, aimed a sensitivity approaching 100 times that of the EVLA, coupled with resolutions from arcseconds to milliarcseconds, could begin construction in 2010 and be completed by the middle of the next decade. Wide-field radio imaging is essentially a solved problem, so for the next five years the main task of the international SKA consortium (who, during this General Assembly, signed a Memorandum of Understanding to participate in the project) is to explore designs which will reduce the cost of collecting area to acceptable levels. When the SKA is realised one can, for the first time, envisage making a radio image of virtually any object found in another waveband.

ALMA is, of course, being constructed in the period up to 2010 and will provide up to 1000 times the sub-mm continuum sensitivity and 50 times the line sensitivity of the present arrays. ALMA is ideally suited for imaging thermal emission with 0".1 -0 ".020 resolution in The Galaxy and high-redshift galaxies.

\subsection{Optical and infrared interferometry}

There are eight or nine interferometer systems currently producing fringes around the world but the study of resolved objects is hard and prospects are comparable to early VLBI images on bright objects (which, one should recall, produced some exciting astrophysics). The generically similar and "semi-industrial" scale programmes at the VLT and KECK sites involve $100 \mathrm{~m}$ to $200 \mathrm{~m}$ baselines and will produce resolving powers of a few milliarcsec in the K-band. Fringe tracking with VLTI should be possible down to an H-band magnitude $\sim 12$ but even this will still leave us barely on the brink of imaging the brightest AGN; the main impact of these programmes will, therefore, be in the study of stellar systems. The Large Binocular Telescope (LBT) programme involves shorter baselines $(24 \mathrm{~m})$ but a much wider field-of-view, while the proposed Large Optical Array (LOA) is aimed squarely at millarcsec resolution.

For mid-ir imaging with resolutions $\sim 0.1$ arcsec the NGST will, of course, be unrivalled but its performance might well be matched, or even exceeded, from the ground in the near-ir and we heard that NGST's launch date has now slipped to 2009 . 


\subsection{Large optical reflectors}

There are several ambitious concepts (e.g. CELT and OWL) being discussed, for construction in the period $2010-2020$, with diameters ranging from $30 \mathrm{~m}$ to $100 \mathrm{~m}$. Such telescopes would exceed the sensitivity of the present $8-10 \mathrm{~m}$ class telescopes by one to two orders of magnitude. These ultra-large telescopes will, however, only achieve their full potential if AO can be made to work for the complete aperture, and if we learn how to deal with the structural deformations produced by turbulent winds. The prize is great: detection of faint objects down to magnitudes in the mid-30s, coupled with resolutions of a few millarcsec. The complementarity with SKA is obvious.

\subsection{High resolution $X$-ray and gamma-ray imaging}

X-ray interferoemtry is almost certainly a long way off but we heard of some proposals which may come to fruition in the working lifetimes of the younger half of the audience. In an ideal world MAXIM \& MAXIM2 could even be the next-but-one US X-ray projects. These concepts offer wonderful prospects for ultra-high X-ray resolution but unsurprisingly there many technological hurdles to overcome. Moving up in frequency, I was surprised to find that concepts for gamma-ray interferometry are already being explored. We can only hope that some of us might live to see the images.

\subsection{Space Astrometry}

The next high resolution steps in space will not involve imaging but will concentrate on astrometry. SIM is due for launch in 2008 and should produce a few microarcsec accuracy on 5000 targets down to 20 mag. FAME, due for launch in 2004, could be dubbed the "son of Hipparcos" and is aimed at collecting data on 40 million targets with $\sim 100$ microarcsec accuracy. GAIA, which might optimistically be launched as early as 2010 , could be dubbed the "grandson of Hipparcos" and is aimed at astrometry of 1000 million objects with $\sim 10 \mathrm{mi}$ croarcsec precision down to $15 \mathrm{mag}$. The success of the Hipparcos mission is evident in its parenthood of future generations!

\section{Some Closing Thoughts}

The new results we heard during this meeting allow us to update three of Martin Rees's "statements" in his opening talk.

Images showing variability are rare: to the original list of three viz. superluminal "blobs" in AGN jets; water maser motions in star forming regions and stellar motions in the Galactic Centre we can now add: expanding (and decelerating) supernova shells, motions of $\mathrm{SiO}$ masers in the photosphere of a red giant star and sub-relativistic flows in the jets of Herbig-Haro objects.

Jets: A new handle on jet physics is the detection of several jets in Xrays by Chandra. While VLBI can't yet resolve details down to gravitationally significant scales it is approaching this limit for the Galactic Centre. The development of sub-mm VLBI and second-generation, space VLBI missions, will allow us probe these scales in nearby AGN such as Cen A and M87. 
Coherent Emission? The IDV and space VLBI results show that many sources exceed the $10^{12}$ inverse-Compton "limit", sometimes by factors of several hundred. Rees pointed out that there are problems with jet stability if the required Lorentz factors for beaming are too high.

Finally, and despite some notable exceptions, one has to conclude that in the year 2000 we still cannot tackle a wide range of astrophysical topics with high resolution observations across the EM spectrum. This disappointing state-of-affairs will, however, change dramatically over the course of the next decade as many new, and much more sensitive, telescopes with resolving powers $\sim 0.1$ arcsec come into operation - to name just a few: EVLA, e-MERLIN, ground-based optical-ir adaptive optics and interferometry, ALMA, NGST, and the continuation of the HST. In the decade following we should see e-VLBI, OWL/CELT, SKA and DARWIN and, possibly, MAXIMA. The flood of new complementary data will surely lead to much greater astrophysical synergies than we saw in this symposium-good news for symposium organisers at the IAU in 2009 and $2012 ! !$ 